\title{
Muscle-Sparing ADM-Assisted Breast Reconstruction Technique Using Complete Breast Implant Coverage: A Dual-Institute UK-Based Experience
}

\author{
Raghavan Vidya $^{\mathrm{a}}$ Simon J. Cawthorn ${ }^{\mathrm{b}}$ \\ ${ }^{a}$ Breast Care Centre, Royal Wolverhampton Hospital NHS Trust, Wolverhampton, United Kingdom; \\ ${ }^{b}$ Breast Care Centre, North Bristol NHS Trust, Southmead Hospital, Bristol, United Kingdom
}

Keywords

Pectoralis muscles · Breast - Mammaplasty .

Acellular dermis · Mastectomy · Breast implants

\section{Summary}

Background: We report our early experience of a novel muscle-sparing breast (prepectoral) reconstruction technique using a pre-shaped Braxon ${ }^{\circledR}$ mesh (acellular dermal matrix) which completely wraps around the breast implant. Methods: All patients who underwent prepectoral implant-based breast reconstruction between April 2014 and September 2015 were included in the analysis. The dermal matrix Braxon ${ }^{\circledR}$ used is a pre-shaped matrix which forms a complete implant mesh wrap. The new breast created is placed over the chest wall without disturbing the pectoralis musculature. Results: A total of 51 (42 unilateral and 9 bilateral) muscle-sparing breast reconstructions were carried out. Complications included implant loss ( $n=1 ; 1.7 \%$ ) secondary to wound infection, seroma ( $n=4 ; 6.7 \%)$, and superficial wound dehiscence ( $n=1 ; 1.7 \%$ ) which was re-sutured without further complication. The median follow-up period was 16.4 (range 8-25) months. Conclusion: The early experience appears highly satisfactory with good clinical outcome. The novel prepectoral implant-based breast reconstruction using the mesh wrap provides an effective alternative to the more traditional submuscular implant-based technique.

Partial data of the present study were collected in the multi-center study of the BIG Group and presented at the Barcelona Breast Meeting (BBM 2015), Barcelona, 11-13 March 2015, and at the ABS Conference \& AGM, Bournemouth International Centre, 15-16 June 2015.

\section{Introduction}

Breast cancer is the most common cancer in the UK, and around 50,000 women are newly diagnosed with the disease each year. In England, 4 in 10 women diagnosed with breast cancer undergo mastectomy as their primary therapeutic procedure. Implant-based breast reconstruction accounts for $37 \%$ of all breast reconstructions performed in the UK [1].

The introduction of biological matrices over the last decade has changed the facet of breast reconstruction. A variety of meshes are available on the market derived from both allogenic and xenogenic donor sources [2-4]. Acellular dermal matrices (ADMs) are the most commonly used biomaterials and can be derived from human, porcine, and bovine sources. The classic technique of implant-based reconstruction is the subpectoral technique which involves recruitment of the pectoralis major muscle. The matrix is placed in the lower pole of the breast, and it forms an internal bra along with the chest wall muscle to hold the definitive implant $[5,6]$. Indeed, 1-stage implant-based breast reconstruction using biological matrix has become very popular in the last 10 years. The advantages of single-stage reconstruction include improved lower pole projection and a better psychological impact due to single surgery $[5,6]$. Despite advantages, the literature reveals conflicting reports, especially in relation to postoperative complications $[7,8]$. Authors have reported higher rates of seroma formation and infection, while others observed no differences in the postoperative complication rates when compared to traditional expander-assisted breast reconstruction [9].

The rapid evolution of new meshes has led to the emergence of prepectoral techniques of breast reconstruction. The muscle-sparing technique of implant-based breast reconstruction using preshaped mesh (Braxon ${ }^{\circledR}$, Decomed S.r.l., Venezia, Italy) has been recently described [10]. Hence, we adopted this novel technique of prepectoral implant-based breast reconstruction in our practice, and herein present our initial experience.

\section{KARGER}

() 2017 S. Karger GmbH, Freiburg 


\section{Patients and Methods}

All patients who underwent muscle-sparing breast reconstruction from April 2014 to September 2015 were included in the analysis. They provided informed consent for use of clinical data, and complete approval of the protocol was obtained from the institutional review committee of each centre.

\section{Patient Selection}

Patients were selected according to the Association of Breast Surgery and the British Association of Plastic, Reconstructive and Aesthetic Surgeons' guidelines for ADM-assisted implant-based breast reconstruction [11]. Inclusion criteria included a body mass index (BMI) of $<35 \mathrm{~kg} / \mathrm{m}^{2}$, no previous radiotherapy, an estimated mastectomy weight of $<600 \mathrm{~g}$, and a good subcutaneous layer (pinch test $>1 \mathrm{~cm}$ ). All patients who needed anticipated postoperative radiotherapy and smokers were excluded.

\section{Technique}

The surgery was carried out by 2 consultant surgeons in each centre. It was ensured that the mastectomy flaps were well vascularized and diathermy use was minimised to preserve the vascularity of the flap, as this promotes integration of the ADM mesh implant wrap.

For complete coverage of the breast implant, a pre-shaped, 0.6-mm thick, porcine ADM was used (fig. 1). The Braxon ${ }^{\circledR}$ matrix, a non-cross-linked ADM of about $30 \times 20 \mathrm{~cm}$, required 5-10 min hydration in saline solution as per the manufacturer's instructions. The patented shape of the matrix fitted both round and anatomical breast implants ranging from 150 to $500 \mathrm{cc}$. The selected implant was placed inside the matrix and completely wrapped by suturing the edges of the matrix together with absorbable stitches (2-0 Vicryl) to form a snug pocket (fig. 1). The new mesh implant wrap was placed onto the pectoralis without detaching it and anchored to the chest wall using apical, medial, and lateral sutures. This ensures fixation of the mesh implant wrap and prevents any rotation. ADM fixation is crucial to ensure primary stability and intimate contact of the matrix with the vascularized tissue, which are the basic requirements that promote remodelling of the collagen membrane $[10,12]$. For this reason, a quilting suture between the ADM and the subcutaneous layer is recommended before closure of the skin. Neovascularization and the inherent capacity of the biomaterial to be incorporated into the surrounding tissues provide a stable permanent cover. 2 vacuum drains ( $12 \mathrm{~F}$ ) were inserted anteriorly and posteriorly; prophylactic antibiotics were administered routinely for 5 days after surgery. Women were discharged from the hospital with their drains in situ as per unit practice. Drains were removed when drainage was less than $20 \mathrm{ml}$ per day over $48 \mathrm{~h}$. All patients were followed-up at 2 weeks, 4 weeks, and 6 months postoperatively.

\section{Results}

A total of 51 women underwent prepectoral breast reconstruction with the Braxon ${ }^{\circledR}$ ADM (table 1). A total of 60 implant-based reconstructions were carried out with $41(82.4 \%)$ unilateral and 9 (17.6\%) bilateral procedures. The majority of patients $(n=44$; $86.3 \%$ ) underwent mastectomy for breast cancer (38 with immediate, 6 with immediate-delayed reconstruction following neoadjuvant chemotherapy), 5 (9.8\%) women had risk-reducing surgery, and $2(3.9 \%)$ underwent revision surgery for capsular contracture following previous submuscular implant-based reconstruction.

The median age was 55 years (range 28-71 years), the mean BMI was $26.4 \mathrm{~kg} / \mathrm{m}^{2}$ (range $20.3-34.8 \mathrm{~kg} / \mathrm{m}^{2}$ ), and the median breast implant weight was $360 \mathrm{~g}$ (range 175-480 g). All patients were non-smokers and had no preoperative radiotherapy. Patients were followed-up for a minimum of 8 months and a maximum of
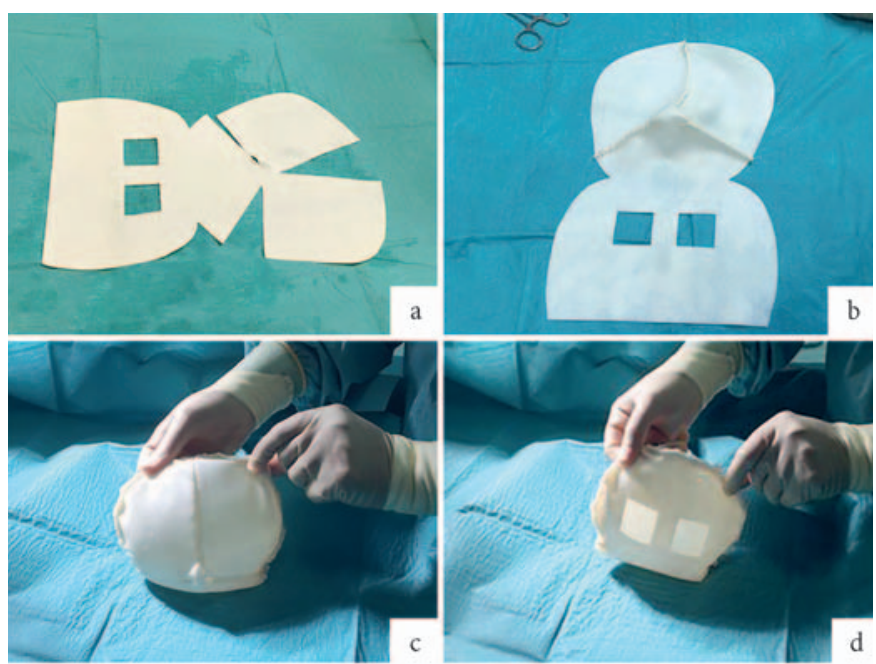

Fig. 1. Breast implant wrapping with pre-shaped acellular dermal matrix (ADM): a Positioning of the hydrated pre-shaped Braxon ${ }^{\circledR} \mathrm{ADM}$ on a sterile table. The windowed part of the matrix (posterior wrap) is design to form the rear portion of the implant wrap, preventing seroma formation; b Suture of the frontal shell of the dermal implant pocket with absorbable suture (2-0 Vicryl); c Positioning of the definitive breast implant in the ADM pocket. Suturing of the frontal and posterior part of Braxon ${ }^{\circledR}$ and trimming of the excess matrix in the lateral borders. Picture shows the frontal part of the wrapped implant; d Posterior part of Braxon with windows to prevent fluid accumulation. This part of the wrapped implant will be placed onto the pectoralis muscle and sutured to the chest wall.

Table 1. Demographic data of the patients

\begin{tabular}{lc}
\hline Characteristics & \\
\hline Patients, $\mathrm{n}$ & 51 \\
Braxon implants, $\mathrm{n}(\%)$ & 60 \\
$\quad$ Total & $41(82.4)$ \\
$\quad$ Unilateral & $9(17.6)$ \\
$\quad$ Bilateral & $44(86.3)$ \\
Mastectomy for breast cancer, n (\%) & $5(9.8)$ \\
Risk reducing surgery, n (\%) & $2(3.9)$ \\
Revision surgery, n (\%) & $1(2.0)$ \\
Postoperative radiotherapy, n (\%) & $55(28-71)$ \\
Median age (range), years & $26.4(20.3-34.8)$ \\
Median body mass index (range), kg/m² & $360(175-480)$ \\
Median size of the implant (range), g & $16.4(8-25)$ \\
Median follow-up (range), months &
\end{tabular}

Table 2. Postoperative complications

\begin{tabular}{ll}
\hline Complications & $\mathrm{n}, \%$ \\
\hline Haematoma & $0(0)$ \\
Red breast syndrome & $0(0)$ \\
Superficial skin necrosis $^{\mathrm{a}}$ & $1(1.7)$ \\
Dehiscence $^{\mathrm{a}}$ & $1(1.7)$ \\
Seroma $^{\mathrm{b}}$ & $4(6.7)$ \\
Implant loss $^{\mathrm{c}}$ & $1(1.7)$ \\
\hline
\end{tabular}

${ }^{\mathrm{a}}$ Conservatively treated.

${ }^{b}$ Resolved by aspiration under ultrasound guidance. ${ }^{e}$ Due to wound infection. 

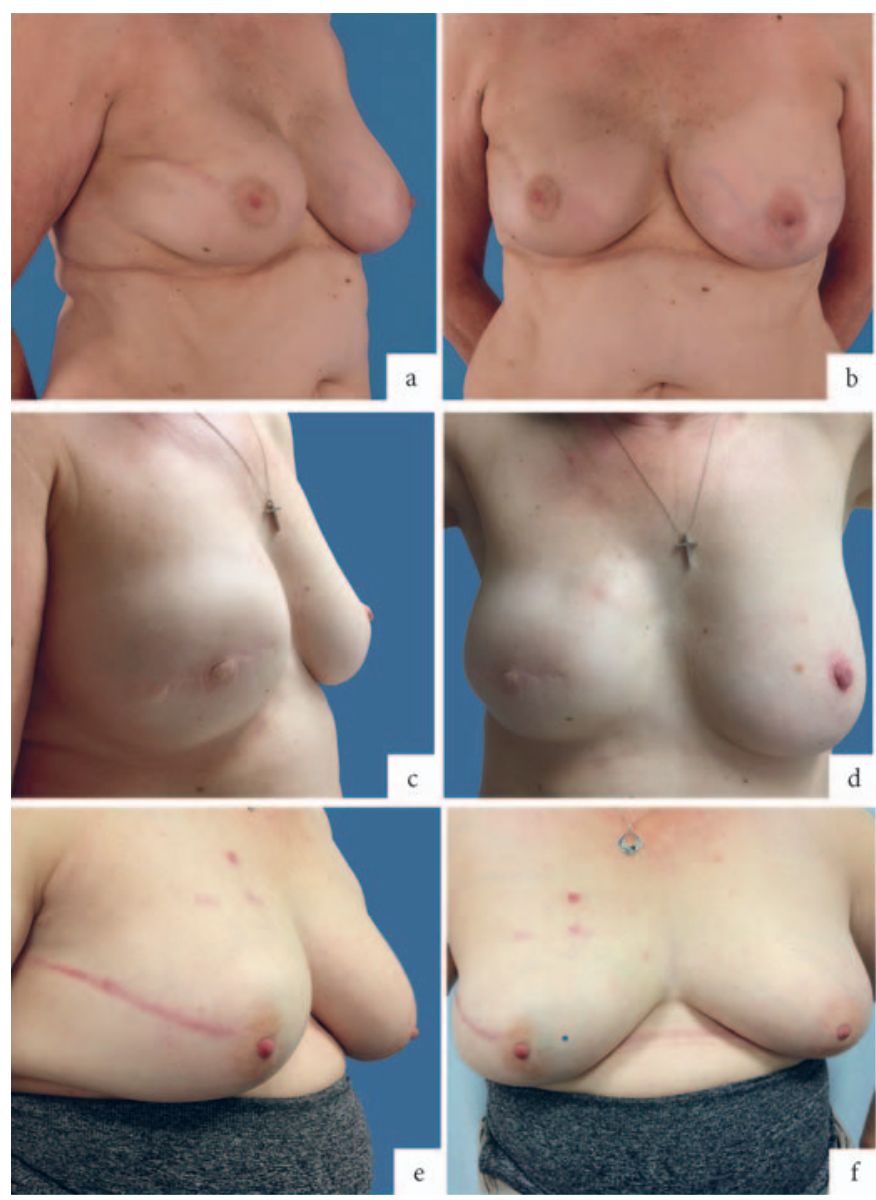

Fig. 2. Aesthetic outcomes: a, b Right prepectoral breast implant with complete acellular dermal matrix (ADM) coverage; 6 months post-operative, frontal and lateral views. Slight asymmetry but patient did not want any contralateral surgery; c, d Right muscle-sparing breast reconstruction with complete breast implant coverage and nipple reconstruction; 11 months post-operative, frontal and lateral views; e, f Right subcutaneous breast reconstruction with complete implant coverage; 7 months post-operative, frontal and lateral views.

25 months (median follow-up 16.4 months). Only 1 patient needed postoperative radiotherapy, and this was well tolerated.

The complications are tabulated in table 2.1 (1.7\%) implant loss occurred in the patient who received neoadjuvant chemotherapy secondary to wound infection 4 weeks after surgery. 4 (6.7\%) breasts developed seroma (2-4 weeks after surgery), successfully treated with ultrasound-guided aspiration. 1 (1.7\%) patient had wound dehiscence, which was re-sutured under local anaesthetic and healed well. 1 (1.7\%) patient had superficial skin necrosis (about $2 \mathrm{~mm}$ ), which resolved with conservative treatment. No red breast syndrome, major bruising, or haematoma formation were observed in our series, which could be as a result of avoiding muscle dissection.

The aesthetic results in terms of shape, softness, and ptosis were highly satisfactory (fig. 2). The reconstructed breasts had natural movement with no dancing breast syndrome or rippling. We also observed clinically that shoulder movement was well-preserved and required minimal analgesia and physiotherapy after the surgery, although no formal objective measures were carried out.

\section{Discussion}

ADMs were first described for use in breast surgery about a decade ago and, since then, have become an important facet of breast reconstruction. ADMs have improved the aesthetic outcomes and reduced the physiological impact of reconstruction by allowing single surgery $[5,6]$. Despite the great advantages, higher postoperative complications have been reported in some series [7, 8], and our single institution experience with submuscular ADM-assisted breast reconstruction revealed $13 \%(n=6)$ implant loss compared with $1.7 \%(\mathrm{n}=1)$ for the prepectoral reconstruction procedure [13]. The literature reveals poor correlation between the onset of postoperative complications and the use of ADM $[14,15]$. A recent review by Clemens et al. [16] proved a positive effect with the use of ADM in settings of irradiated breast tissue; it does not increase or decrease the risk of complications but instead provides psychological and aesthetic benefits.

The major problem related to the submuscular techniques seems to be attributed to the detachment of the pectoralis major. Literature confirms that the weakening of the muscle can alter the function of the shoulder joint and significantly impact activities of daily living $[17,18]$. In our study, we observed clinically that shoulder movement was well preserved and postoperative analgesic requirements were minimal; however, these findings are limited due to a lack of objective assessment. Thus, the new muscle-sparing reconstructive technique appears to enhance faster patient recovery with less postoperative pain and early return to normal activity, as the preservation of the pectoralis major muscle maintains the functionality of the shoulder. This early observation needs to be confirmed by objective assessment in future studies.

Our initial experience with the Braxon ${ }^{\circledR} \mathrm{ADM}$, which allows a subcutaneous implant-based breast reconstruction, appears to be highly satisfactory. We observed in our study a low rate of complications, particularly implant loss (1.7\%) and seroma formation (6.7\%), while the literature review following ADM-assisted breast reconstruction reveals rates of implant loss of $6-15.4 \%$ and seroma formation of 2-19.2\% [8]. The multicentre European study, using Braxon ${ }^{\circledR}$ in 100 cases (median follow-up 17.9 months), revealed only $2 \%$ implant loss, $1 \%$ wound breakdown, $5 \%$ seroma formation, and $2 \%$ haematoma formation [19]. The good outcome with low complication rates can be attributed to case selection (no major comorbidity) and adoption of meticulous surgical technique carried out by experienced consultant breast surgeons.

From a surgeon's perspective, the muscle-sparing breast reconstruction technique with Braxon ${ }^{\circledR} \mathrm{ADM}$ had a short learning curve and reduced operating time as the creation of the ADM breast implant pocket is simple, fast, and easy to learn. Moreover, patients with a muscle-sparing reconstruction did not demonstrate any muscle impairment due to the lack of subpectoral dissection, although no formal objective measures were carried out.

Revascularization and the inherent capacity of the ADM to be incorporated into surrounding tissues provide a stable permanent cover $[10,20,21]$. The anchorage of the wrapped breast implant to the pectoralis major muscle and the subcutaneous tissue is essential 
to obtain intimate contact between the ADM and the living tissue in order to promote vascularization and the remodelling process [22]. We advocate that the matrix must form a snug pocket around the implant and be anchored to the subcutaneous tissue. This would promote the incorporation of the matrix implant and reduce seroma formation [22].

It has been postulated that complete mesh wrap may reduce capsular contracture as ADM-based breast reconstruction has shown to have a lower incidence of capsular contracture [23]. Orenstein et al. [24] demonstrated that in vitro ADM inhibits the production of interleukin and vascular endothelial growth factor which may explain this, and in a recent case we reported the integration of Braxon ${ }^{\circledR}$ matrix in human tissue 12 months after implantation, supporting the concept of a complete breast implant coverage made with ADM [22]. Whilst this technique may reduce capsular contracture, only observational relationships can be concluded. In addition, the short-term follow-up further restricts any verification.

\section{Conclusion}

Our early experience using the pre-shaped Braxon ${ }^{\circledR}$ mesh appears to be highly satisfactory. Indeed, the prepectoral technique appears to be a novel alternative in implant-based breast reconstruction. However, further studies looking at long-term outcomes are still required.

\section{Disclosure Statement}

The authors declare no conflict of interest nor financial or personal relationships with anybody that could influence the study. No grant has been received for this study.

\section{References}

1 Jeevan R, Cromwell D, Browne J, et al.: The National Mastectomy and Breast Reconstruction Audit. A national audit of provision and outcomes of mastectomy and breast reconstruction surgery for women in England. Second Annual Report, 2009.

2 Ibrahim AM, Koolen PG, Ashraf AA, Kim K, Mureau MA, Lee BT, Lin SJ: Acellular dermal matrix in reconstructive breast surgery: survey of current practice among plastic surgeons. Plast Reconstr Surg Glob Open 2015;3:e381

3 Gandhi A, Barr L, Johnson R: Bioprosthetics: changing the landscape for breast reconstruction? Eur J Surg Oncol 2013;39:24-25.

4 Macadam SA, Lennox PA: Acellular dermal matrices: use in reconstructive and aesthetic breast surgery. Can J Plast Surg 2012;20:75-89.

5 Sbitany H, Langstein HN: Acellular dermal matrix in primary breast reconstruction. Aesthet Surg J 2011, 31(suppl 7):30S-37S

6 Breuing K, Colwell A: Inferolateral AlloDerm ham mock for implant coverage in breast reconstruction Ann Plast Surg 2007;59:250-255

7 Sbitany H, Serletti JM: Acellular dermis-assisted prosthetic breast reconstruction: a systematic and critical review of efficacy and associated morbidity. Plast Reconstr Surg 2011;128:1162-1169.

8 Hoppe IC, Yueh JH, Wei CH, Ahuja NK, Patel PP, Datiashvili RO: Complications following expander/implant breast reconstruction utilizing acellular dermal matrix: a systematic review and meta-analysis. Eplasty 2011:11:e40

9 Davila AA, Seth AK, Wang E, Hanwright P, Bilimoria K, Fine N, Kim JY: Human acellular dermis versus submuscular tissue expander breast reconstruction: a multivariate analysis of short-term complications. Arch Plast Surg 2013;40:19-27.
10 Berna G, Cawthorn SJ, Papaccio G, Balestrieri N: Evaluation of a novel breast reconstruction technique using the Braxon ${ }^{\circledR}$ acellular dermal matrix: a new musclesparing breast reconstruction. ANZ J Surg 2017;87: 493-498.

11 Martin L, O’Donoghue JM, Horgan K, Thrush S, Johnson R, Gandhi A; Association of Breast Surgery and the British Association of Plastic, Reconstructive and Aesthetic Surgeons: Acellular dermal matrix (ADM) assisted breast reconstruction procedures: joint guidelines from the Association of Breast Surgery and the British Association of Plastic, Reconstructive and Aesthetic Surgeons. Eur J Surg Oncol 2013;39:425-429.

12 Nahabedian MY: Acellular dermal matrices in primary breast reconstruction: principles, concepts, and indications. Plast Reconstr Surg 2012;130:44S-53S.

13 Potter S, Chambers A, Govindajulu S, Sahu A, Warr R, Cawthorn S: Early complications and implant loss in implant-based breast reconstruction with and without acellular dermal matrix (Tecnoss Protexa ${ }^{\circledR}$ ): a comparative study. Eur J Surg Oncol 2015;41:113-119.

$14 \mathrm{Vu}$ MM, Kim JY: Current opinions on indications and algorithms for acellular dermal matrix use in primary prosthetic breast reconstruction. Gland Surg 2015;4: 195-203.

15 Lardi AM, Ho-Asjoe M, Mohanna PN, Farhadi J: Immediate breast reconstruction with acellular dermal matrix: factors affecting outcome. J Plast Reconstr Aesthet Surg 2014;67:1098-1105.

16 Clemens MW, Kronowitz SJ: Acellular dermal matrix in irradiated tissue expander/implant-based breast reconstruction: evidence-based review. Plast Reconstr Surg 2012;130(suppl 2):27S-34S.
17 Wallace MS, Wallace AM, Lee J, Dobke MK: Pain after breast surgery: a survey of 282 women. Pain 1996;66 195-205.

18 Kiernan T, Martin L: Use of acellular dermal matrix is comparable to expander based breast reconstructions for post-operative physiotherapy requirements. Surgery Curr Res 2013;3:136-137.

19 Vidya R, Masia J, Berna G, Dell'Antonia F, Cawthorn S, Bozza F, Gardetto A, Kolacinska A, Tiengo C, Bassetto F, Caputo GG, Governa M: Evaluation of the effectiveness of the prepectoral breast reconstruction with Braxon dermal matrix: first multicentre European report on 100 cases. Breast J 2017;Epub ahead of print.

20 Cheng A, Lakhiani C, Saint-Cyr M: Treatment of capsular contracture using complete implant coverage by acellular dermal matrix: a novel technique. Plast Reconstr Surg 2013;132:519-529.

21 Reitsamer R, Peintinger F: Prepectoral implant placement and complete coverage with porcine acellular dermal matrix: a new technique for direct-to-implant breast reconstruction after nipple-sparing mastectomy. J Plast Reconstr Aesthet Surg 2015;68:162-167.

22 Iqbal FM, Bhatnagar A, Vidya R: Host integration of an acellular dermal matrix: Braxon mesh in breast reconstruction. Clin Breast Cancer 2016;16:e209-e211.

23 Mofid MM: Acellular dermal matrix in cosmetic breast procedures and capsular contracture. Aesthet Surg J 2011;31(suppl 7):77S-84S

24 Orenstein SB, Qiao Y, Kaur M, Klueh U, Kreutzer DL Novitsky YW: Human monocyte activation by biologic and biodegradable meshes in vitro. Surg Endosc 2010; 24:805-811 